\title{
Two Rare Cases of Bronchus-Associated Lymphoid Tissue Lymphoma Successfully Treated with Rituximab-Bendamustine
}

\author{
Nydia Panitz ${ }^{1}$, Kristin Kuepper ${ }^{1}$, Cornelia Becker ${ }^{2}$, Hendrikje Schleife ${ }^{3}$, Enrica Bach ${ }^{1}$, \\ Sabine Opitz ${ }^{1}$, Alexander Schaudinn ${ }^{1}$, Uwe Platzbecker ${ }^{1}$, and Sabine Kayser ${ }^{4}$ \\ ${ }^{1}$ Univerversity Hospital Leipzig \\ ${ }^{2}$ Onkologie Praxis Leipzig \\ ${ }^{3}$ Onkologie Praxis Frohburg \\ ${ }^{4}$ DKFZ
}

May 3, 2021

\begin{abstract}
The bronchus-associated lymphoid tissue (BALT) lymphoma is a rare subtype of extranodal marginal zone lymphoma of mucosaassociated lymphoid tissue (MALT lymphoma), affecting roughly $9 \%$ of all MALT lymphomas. The underlying pathomechanism of BALT lymphomas is not completely resolved and a standard of care treatment is so far not available.
\end{abstract}

\section{Introduction}

Extranodal marginal zone lymphoma of mucosa-associated lymphoid tissue lymphomas (MALT lymphoma) belongs to mature B-cell neoplasms and thus to the huge class of non-Hodgkin's lymphoma (NHL). MALT lymphoma account for 7-8\% of B-cell lymphomas, most commonly affecting the stomach (35\%), ocular adnexa (13\%), skin $(9 \%)$, lungs $(9 \%)$, salivary glands $(8 \%)$, breasts $(3 \%)$ and thyroid $(2 \%)$.

The bronchus-associated lymphoid tissue lymphoma (BALT lymphoma) represents the most common histologic subtype of primary pulmonary lymphomas (PPL) with $77-87 \%$. However, PPLs are rare with an occurrence of $0.5-1 \%$ of all pulmonary tumors. Causes to develop a BALT lymphoma are various and not completely understood. In many MALT lymphomas, there is a history of chronic inflammatory disease resulting in the accumulation of extranodal lymphoid tissue. The chronic inflammation may be the result of infection, autoimmunity, smoking, environmental or other unknown risk factors.

Patients are mostly asymptomatic at diagnosis and if symptoms are present, they are rather nonspecific including dyspnea, cough, or B-symptoms for several weeks or months. For diagnosis clinical, medical imaging, histologic and biologic parameters are needed. BALT lymphomas progress slowly and mostly remain within the lung for a long time. Besides treatment/eradication of an underlying chronic inflammatory disorder/trigger factor combined chemo- and/or immunotherapy, surgery and/or local radiation are possible treatment approches. Currently, no standard treatment approach is available partially due to its rare occurrence and heterogeneity. Nevertheless, BALT lymphomas are associated with a favorable prognosis and a 5-year overall survival (OS) rate of $80 \%$ as well as median survival over 10 years.

Here, we report two patients with nonspecific symptoms and blood count anomalies, who were diagnosed with BALT lymphoma in our hospital and treated with a combined immune-chemotherapy of rituximab and bendamustine.

\section{Patient Information}




\section{Case report - patient 1:}

A 58-year-old male patient (ex-smoker, abstinent since 30 years) was admitted in May 2020 to our institution due to very severe thrombocytopenia with platelet counts of $1 \times 10^{9} / \mathrm{L}$ (range, $140-360 \times 10^{9} / \mathrm{L}$ ). The patient reported progressive petechia at the lower legs and large hematomas after minor trauma. A few days prior to admission a dental operation was performed with increased bleeding. Thus, complete blood count was taken. With the exception of extremely low platelets, blood count was unremarkable. No B-symptoms were present.

In suspicion of an idiopathic thromobocytopenic purpura (ITP) therapy with corticosteroids (dexamethasone $40 \mathrm{mg} / \mathrm{kg} /$ body weight for four days) and immunoglobulins $30 \mathrm{~g}$ per day for five days was initiated. Since severe thrombocytopenia was persistent despite combined therapy the thrombopoetin-receptor agonist eltrombopag 50mg/day for 7 days was added. However, there was still no response to therapy.

Flow cytometry analysis of the peripheral blood showed no evidence of a T-cell lymphoma, but a shifted kappa/lambda ratio (0.2) with no reliable differentiation of a monoclonal subpopulation. Bone marrow evaluation displayed a hyperplastic, slightly dysplastic medullary pattern after administration of corticosteroids, but no clear lymphocytic infiltration. Flow cytometry of the bone marrow detected a small monoclonal B-cell population $(0.8 \%)$, most compatible with marginal zone lymphoma, which could not be confirmed by immunohistochemistry staining. Cytogenetics was normal in 20 metaphases. In fluorescence in-situ hybridization analysis no MALT1-rearrangement was present. In addition, a computer tomography-scan (CT) was performed, which showed large, centrally located focal infiltrates in both sides of the lung (Figure 1, Panels A and C) as well as a splenomegaly of $16 \mathrm{~cm}$. Histopathology evaluation after bronchoscopic biopsy revealed a BALT-Lymphoma (Figure 2). Further staging with esophageal-gastro-duodenoscopy detected a gastric infiltration of a MALT-lymphoma without Helicobacter pylori infection resulting in an Ann Arbor stadium IIIA.

A combined immunochemotherapy with rituximab $\left(375 \mathrm{mg} / \mathrm{m}^{2}\right.$, day 0$)$ and bendamustine $\left(90 \mathrm{mg} / \mathrm{m}^{2}\right.$, day 1-2) was initiated. Already in response to rituximab the platelet count increased to $121 \mathrm{x} 10^{9} / \mathrm{L}$. Thus, the patient was discharged and the therapy was continued on an outpatient basis for a total of six cycles, repeated every four weeks. Contrast-enhanced CT stagings were performed after three and six cycles showing no evidence of the disease after six cycles (Figure 1, Panels B and D). Platelets were within normal range during treatment without additional supportive medication or transfusions. In addition, a gastroscopy was performed without any further detection of suspicious results. Thus, complete remission (CR) of the BALT lymphoma was achieved.

\section{Case report - Patient 2:}

A 52-year-old male patient ex-smoker since 3 months (cumulative 30 pack/years) was admitted to our hospital in June 2020 presenting with dry cough since January 2020 and restricted physical resilience including dyspnea since April 2020. He denied B-symptoms. The laboratory evaluation revealed an isolated mild anemia with hemoglobin value of $11.4 \mathrm{~g} / \mathrm{dL}$ (range, 13.5-17.5 g/dl) and an elevated C-reactive protein (CRP) of $86.45 \mathrm{mg} / \mathrm{L}$ (range, $<5 \mathrm{mg} / \mathrm{l}$. Chest X-ray showed bilateral infiltrates, particularly in the left lower lung. An antibiotic therapy with piperacillin/tazobactam was initiated with a decline of the CRP to $16.48 \mathrm{mg} / \mathrm{L}$ after 10 days of therapy. The first bronchoscopy with transbronchial biopsy revealed a highly florid and erosive inflammation with high-grade regenerative hyperplasia of the epithelium, which was CD20 and CD3 positive. The molecular clonality analysis detected immunoglobulin gene rearrangements of IGHA, IGHB and IGHC in 41, 278 and 146 base pairs, respectively. The subsequent CT scan showed still existing bi-pulmonary solid infiltrates in all lobes of the lung (Figure 3, Panels A and C). The suspicion of a BALT lymphoma was substantiated by further bronchoscopy with histopathology analysis showing a sub-mucosal stroma with B-cell containing infiltrates without a specific immunophenotype. A CT-based pulmonary biopsy confirmed a CD20 positive B-cell population without co-expression of CD5, BCL-2, CD10 or CD23 (Figure 4). Based on these results staging analysis containing bone marrow evaluation and extended CT scan were performed. Bone marrow histology showed no lymphatic infiltration. CT scan showed paraaortic, 
iliac and inguinal affected lymph nodes resulting in an Ann Arbor stadium IIIA.

A combined immunochemotherapy containing rituximab $\left(375 \mathrm{mg} / \mathrm{m}^{2}\right.$, day 0$)$ and bendamustine $\left(90 \mathrm{mg} / \mathrm{m}^{2}\right.$, day 1-2) was initiated. The first cycle was well tolerated under standard supportive care without any serious adverse events or allergic reactions. During the hospitalization, the patient developed hypoxia at rest. Thus, he received oxygen up to $4 \mathrm{~L} / \mathrm{h}$ via nose prongs. After the first cycle of immunochemotherapy, the unproductive cough improved and the oxygen therapy could be stopped. The immunochemotherapy was repeated every four weeks for in total six cycles; CT stagings were performed after three and six cycles. The intermediate CT scan showed no significant changes of the BALT lymphoma and the affected lymph nodes as compared to diagnosis. However, the clinical condition improved significantly. Therefore, the immunochemotherapy was continued. After six cycles a native CT scan was performed showing a reduction of the BALT lymphoma and normalization of the lymph nodes. Unfortunately, the patient denied a positron emission tomography-CT. According to the CT scan at least a partial remission (PR) was achieved (Figure 3, Panels B and D). Thus, maintenance therapy with rituximab is planned.

\section{Discussion}

The BALT lymphoma is a rare hematologic entity with less than $1 \%$ occurrence of all pulmonary tumors, but it represents the most common histologic subtype in all primary pulmonary lymphomas. The pathology and genetics are not completely deciphered, however, prognosis and 5-year OS rate with $85 \%$ are favorable. The BALT lymphoma is not easy to diagnose because of its rarity, heterogeneity and nonspecific symptoms as well as often oligo- or asymptomatic patients. In our case, one patient developed dry cough and exertional dyspnea over few months without further symptoms. In line with previous reports CT-scan showed solitary pulmonary nodules in all lung lobes. Based on the CT scan, a biopsy of lung tissue, clonality and immunohistochemistry analysis were performed as recommended (NCI SEER database). The clonality analysis detected immunoglobulin gene rearrangements in IGHA, IGHB and IGHC, which have previously been described to be affected in BALT lymphomas. The immunostaining of the lung tissue identified CD20 positive B-cells in the infiltrate without any co-expression of CD5, CD10, CD23, BCL6 or BCL2, compatible with a BALT lymphoma. Most of the patients are diagnosed with stage I or II disease, but roughly $40 \%$ show involvement of multiple extranodal sites. Staging in patients with multiple extranodal lesions may be challenging, since at least some cases constitute multiple clonally unrelated proliferations rather than truly disseminated disease.

For the diagnosis of a BALT lymphoma various methods including radiologic imaging, histology and genetic analysis are needed. Additional investigations should also be taken into account such as gastroscopy to identify a possible involvement of other organ systems. In our cases, one patient showed paraaortic, iliac and inguinally enlarged lymph nodes and the other patient presented with splenomegaly as well as a gastric manifestation.

The treatment of BALT lymphoma is not well standardized due to the rarity and heterogeneity of involved sites. Indeed, many therapeutic options are available including surgery, radiotherapy, immunotherapy and chemotherapy. In many MALT lymphoma patients, there is a history of a chronic inflammatory disorder resulting in the accumulation of extranodal lymphoid tissue. The chronic inflammation may be the result of an infection, autoimmunity or other unknown stimuli. This link is most clearly established for Helicobacter pylori and gastric MALT lymhoma. The importance of this stimulation in vivo has been clearly demonstrated by the induction of remissions in gastric MALT lymphomas with antibiotic treatment to eradicate Helicobacter pylori. A role for antigenic stimulation by Chlamydia psittaci and Borrelia burgdorferi has been proposed for some cases of ocular adnexal and cutaneous MALT lymphomas, respectively. A similar role has been proposed for Campylobacter infection in patients with heavy chain disease. The eradication of Helicobacter pylori associated with gastric or Chlamydia psittaci causing ocular adnexia MALT lymphoma by antibiotic therapy led to protracted remissions. Thus, successful eradication or removal of suspected trigger factors e.g. smoking should be of utmost priority.

If BALT lymphoma is localized and accessible, surgery is also a possible treatment option compared to 
chemotherapy. Our patients showed bi-pulmonary and extrapulmonary manifestations. Therefore, local surgery was not an option and an immunochemotherapy with rituximab and bendamustine was performed. A large, prospective randomized multicenter phase III study including over 500 patients with indolent lymphomas including MALT lymphomas and mantle-cell lymphomas showed an increased progression-free survival and fewer toxic effects of rituximab/bendamustine as compared to rituximab, cyclophosphamide, vincristine, doxorubicin and prednisolone (R-CHOP). In addition, Salar et al. showed in a multicenter, single-arm, non-randomized, phase 2 trial on 60 patients with MALT lymphoma at any site and stage a progression-free survival after 7-years of $92.8 \%$. Combinations of the regimen with other therapeutics including the bruton's tyrosine kinase inhibitor PCI-32765, idelalisib, or the PI3Ko inhibitor IBI376 are also investigated in different clinical trials (ClinicalTrials.gov: NCT01479842, NCT03424122). Further clinical studies are evaluating other biologicals, such as obinutuzumab (ClinicalTrials.gov: NCT03322865), venetoclax (ClinicalTrials.gov: NCT04447716) or lenalidomide (ClinicalTrials.gov: NCT03015896, NCT04604028). In previous case reports and studies, rituximab as single agent or combined therapy with chlorambucil, cladribine or CHOP led to good responses with long-term survival up to $70 \%$. Finally, radiation and eradication therapies are also investigated (ClinicalTrials.gov: NCT01820910, NCT03680586, NCT02494700).

Due to previous publications, case reports and our observations, rituximab/bendamustine represents an efficient and well tolerated first-line therapy for MALT lymphoma. Given the safety, tolerability and efficacy we would recommend this immunochemotherapy as first-line therapy, if other trigger factors can be excluded.

Nevertheless, further data, ideally within prospective clinical trials, are required to evaluate the efficacy and safety of immunochemotherapies in BALT lymphoma for various patient collectives. Based on the data from clinical trials future directions for an approved standard treatment with more targeted agents seem to be on the horizon.

\section{Conclusion}

BALT lymphoma is a rare B-NHL with favorable prognosis. We here report on two patients with nonspecific symptoms: one showed as major symptom severe thrombocytopenia and the other dyspnea and dry cough, thereby suggesting an inflammatory focus in the lungs. There is no standard of care established yet. Both of our patients received an immunochemotherapy with rituximab/bendamustine. In previous studies and case reports, rituximab showed promising results and is commonly used as single agent treatment or in combination with chlorambucil or bendamustine. In our patients therapy with rituximab/bendamustine led to promising responses. Thus, we would recommend this immunochemotherapy as first-line therapy as in other MALT lymphomas, if an underlying chronic inflammatory disorder/trigger factor can be excluded. Nevertheless, more investigations and studies are necessary to unravel the underlying pathomechanism of BALT lymphomas, thus enabling to establish a standard of care.

\section{Figure legends:}

\section{Figure 1: Computer tomography scan of the chest of the first patient.}

Panels A) and C) represent the coronal reconstructive and axial section of the chest before start of treatment showing pulmonary focal consolidations centrally pronounced in the entire lung.

Panels B) and D) show the coronal reconstructive and axial section of the patient after six cycles of rituximab and bendamustin, showing a complete remission.

\section{Figure 2: Immunohistochemistry of bronchoscopic biopsy of the first patient.}

Panel A) Infiltration of small cellular lymphoid cell components indicating manifestation of lymphoma in hematoxylin and eosin stain.

Panel B) Anti-CD20 immunohistochemistry confirmed a highly suspicious B cell population.

Panel C) Low proliferation rate of the B cell population detected by MIB-1 immunohistochemistry.

Figure 3: Computer tomography scan of the chest of the second patient. 
Panels A) and C) represent the coronal reconstructive and axial section of the chest before start of treatment showing bi-pulmonary consolidations.

Panels B) and D) show the coronal reconstructive and axial section of the patient after six cycles of rituximab and bendamustine showing a significant reduction in size of pulmonary consolidations (at least partial remission).

Figure 4: Immunohistochemistry of the transbronchial biopsy from the second patient.

Panel A) Infiltration of small cellular lymphoid cell components in the center of the sample in hematoxylin and eosin staining; scale $200 \mu \mathrm{m}$

Panels B) and C): CD20 positivity of the lymphoid infiltrates detected in hematoxylin and eosin staining. B) scale $500 \mu \mathrm{m}$; C) scale $100 \mu \mathrm{m}$

\section{Hosted file}

Figures.pptx available at https://authorea.com/users/411606/articles/520583-two-rare-casesof-bronchus-associated-lymphoid-tissue-lymphoma-successfully-treated-with-rituximabbendamustine 